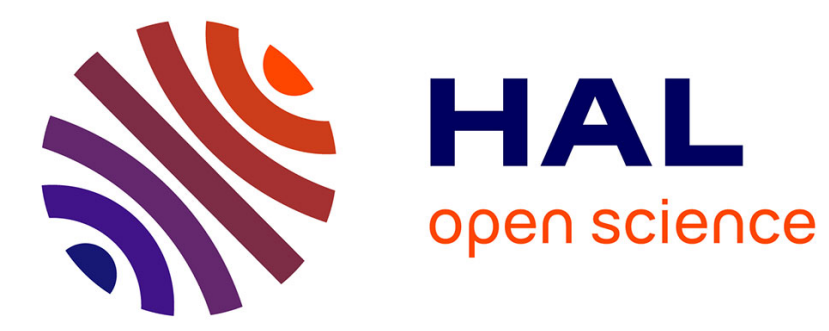

\title{
Le SIG comme outil de détection et d'analyse des parcellaires antiques
}

Maxime Seguin

\section{To cite this version:}

Maxime Seguin. Le SIG comme outil de détection et d'analyse des parcellaires antiques. 3rd International Landscape Archaeology Conference 2014, Sep 2014, Rome, Italie. 10.5463/lac.2014.18 . hal-01487029

\section{HAL Id: hal-01487029 https://hal-inrap.archives-ouvertes.fr/hal-01487029}

Submitted on 10 Mar 2017

HAL is a multi-disciplinary open access archive for the deposit and dissemination of scientific research documents, whether they are published or not. The documents may come from teaching and research institutions in France or abroad, or from public or private research centers.
L'archive ouverte pluridisciplinaire HAL, est destinée au dépôt et à la diffusion de documents scientifiques de niveau recherche, publiés ou non, émanant des établissements d'enseignement et de recherche français ou étrangers, des laboratoires publics ou privés.

\section{(이) $\$$}

Distributed under a Creative Commons Attribution - NonCommercial - NoDerivatives| 4.0 


\title{
Le SIG comme outil de détection et d'analyse des parcellaires antiques
}

\author{
Maxime Seguin \\ Institut National de Recherches Archéologiques Préventives \\ email: maxime.seguin@inrap.fr
}

\section{Abstract}

The French Rhône valley has traces of ancient roman parcels, testified by antique land registers called "Orange cadastre". This organisation was highlighted on the left bank of the river and it seems to extend to the other side, in the Tave and Cèze valleys. All this research was conducted in the 80 s from the French National Institute of Cartography (IGN) aerial missions using the method of optical filtering. In this study, we intend to use a GIS and the cadastral map in vector format (BDParcellaire ${ }^{\circledR}$ product from IGN).

Indeed, the cadastre enables the study of boundaries that have no materialisation in the current landscape. The aerial photographs or topographical maps only give incomplete information, i.e. the perennial human forms or roads, paths, fences, channels, ditches. These documents are selective. The tool used in this study is the histogram of directions. It consists in calculating the azimuth of each parcel boundary and drawing a histogram. The number of boundaries or their total length is shown as a function of the azimuth.

The interests of working with the cadastre are that all information are contained in these documents, their precision allows a detailed study of cadastral frames. This method was first validated on previously studied areas and then extended to the Cèze and Tave lower valleys. This helped to detect the presence of "Orange cadastre" in these two valleys, where the method by optical filtering had not delivered convincing results. The use of these vector data for the detection of ancient cadastral frames permits a detailed study of the boundaries structure. The results are encouraging and allow the consideration of new approaches.
It would be interesting to combine elevation and topographic data as well as ancient "Napoleonic cadastre" with the aim to eliminate nineteenth and twentieth century boundaries.

Keywords: GIS, Land Registers, Histogram of Directions, Vector Cadastral Map, Orange Cadastre

\section{Introduction}

Cet article présente les résultats de travaux de recherches débutés dans le cadre d'un mémoire de fin d'étude à l'Ecole Supérieure des Géomètres Topographes (Seguin, 2010). Ces travaux se sont déroulés dans le cadre du PCR (Projet Collectif de Recherche): Des garrigues au Rhône, occupation du sol et dynamiques de peuplement dans le nord-est du Gard (Alix, 2008) dirigé par Stéphane Alix (Inrap, UMR 6249 Laboratoire de chrono-environnement). Ce PCR a pour objectif l'étude diachronique du peuplement et de l'occupation du sol dans le nordest du département du Gard et plus précisément dans les vallées de la Tave et de la Cèze (fig. 1). Ces deux vallées sont situées entre Uzès et le Rhône, et entre les garrigues au nord de la vallée du Gardon et les collines qui bordent le nord de la vallée de la Cèze. Le principal objectif est de rassembler et d'homogénéiser les données et d'en tirer une synthèse concernant les dynamiques de peuplement pour chaque période chronologique. Ces périodes s'étendent du Néolithique jusqu'à la fin du Moyen-Âge.

Le paysage de la vallée du Rhône comporte des traces de parcellaires antiques attestés par les cadastres antiques dits "d'Orange", gravés dans le marbre. Cette trame parcellaire s'étend 


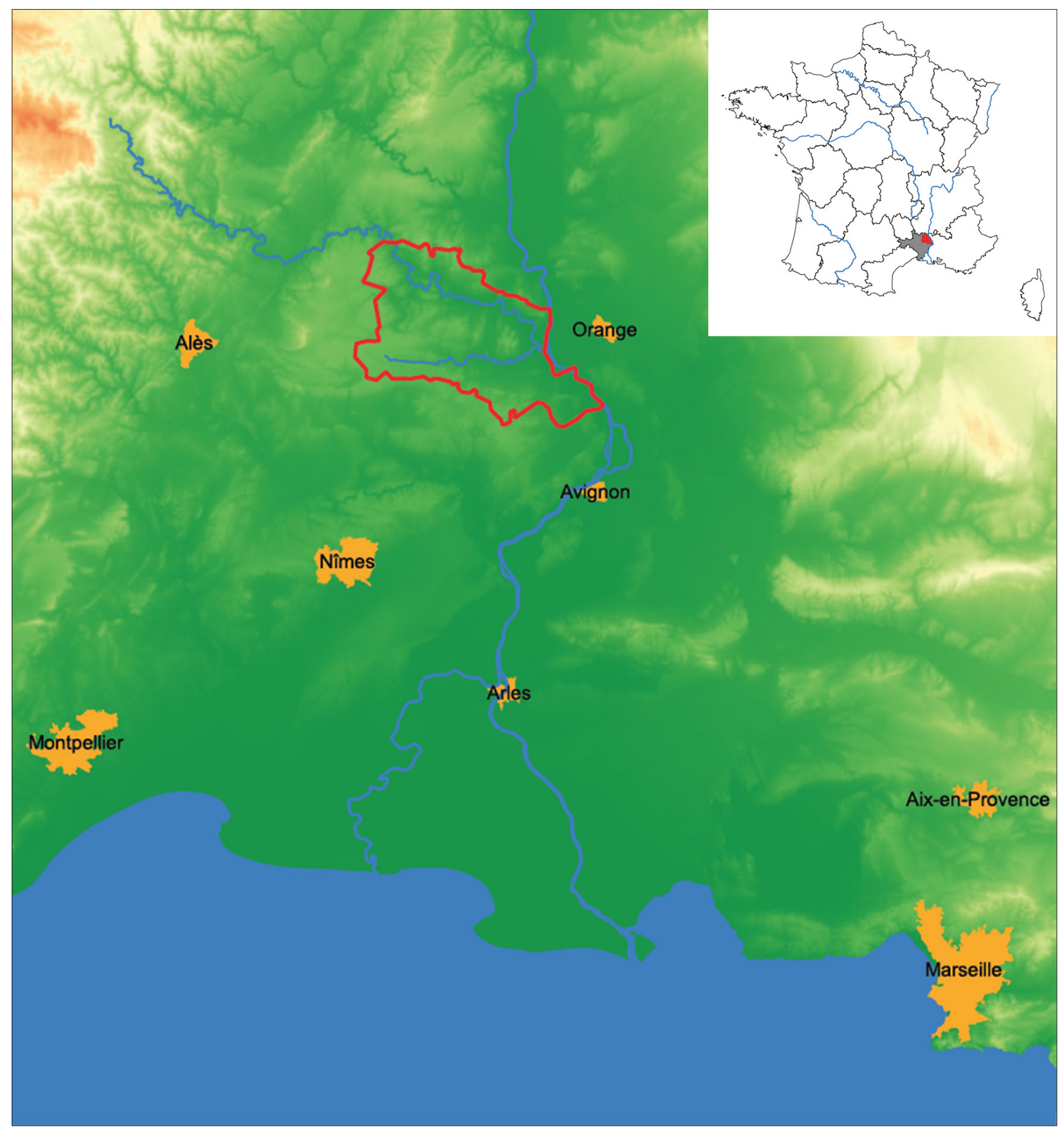

Fig. 1. Localisation des vallées de la Cèze et de la Tave (BDAlti®, CIGN, Inrap, M. Seguin).

le plan cadastral numérique (BDParcellaire ${ }^{\circledR}$ ) à l'aide d'un Système d'Information Géographique (SIG).

En effet, le cadastre permet d'étudier les li-

plus ou moins à la vallée de la Tave et de la Cèze. L'ensemble des recherches sur la vallée du Rhône a été effectuée à l'aide de photographies des missions aériennes de l'Institut Géographique National (IGN), par le procédé de filtrage optique. Dans le cadre de cet article on se propose d'exploiter mites parcellaires qui n'ont plus de matérialisation dans le paysage actuel. Les photographies aériennes ou les cartes topographiques ne restituent qu'une partie de l'information, c'est-à-dire uniquement les formes anthropiques pérennes: routes, 
chemins, clôtures, canaux, fossés. Ce sont des documents sélectifs qui permettent une analyse archéomorphologique basée sur les axes structurants du paysage. L'intérêt de travailler avec le cadastre est de disposer d'un document riche en informations parcellaires, mais qui est composé de traces dynamiques dont la variabilité peut être importante.

L'étude s'est pour l'instant limitée aux communes les plus proches du Rhône: Bagnols-surCèze, Chusclan, Orsan, Codolet, Laudun-l'Ardoise, Saint-Victor-la-Coste, Saint-Geniès-de-Comolas, Montfaucon et Roquemaure.

\section{Les cadastres d'Orange}

Les cadastres antiques sont caractérisés par un réseau d'unités carrées ou rectangulaires. L'implantation dans le paysage de ce genre de trame est donc essentiellement limitée aux grandes plaines alluviales. Les superficies des cadastres peuvent varier de quelques dizaines d'hectares à plusieurs centaines d'hectares. La trame est formée par le croisement d'axes parallèles. On a donc affaire à une structure régulière et géométrique. Dans le cas des cadastres d'Orange, les cadastres $B, C$ et $D$ sont basés sur des unités carrées alors que le cadastre $A$ possède une trame rectangulaire. La logique de la trame orthogonale est conservée jusque dans le découpage des parcelles.

Au XIXe siècle des fragments de marbres sont découverts dans le théâtre d'Orange et dans les années 1950 deux fouilles majeures mettront au jour un nombre important de fragments. André Piganiol publiera en 1962 l'ensemble des fragments découverts (Piganiol, 1962). Il identifiera trois plans cadastraux et les nommeras A, B et $C$. Ces codes sont toujours utilisés pour les désigner. Le cadastre B reste le mieux connu et ce sera le premier à pouvoir être recalé sur le paysage actuel. Après plusieurs études il s'avérera que ces cadastres furent dressés autour de 77 après J.C. sous le règne de Vespasien, bien après la matérialisation physique de la trame dans le paysage rhodanien.

\section{Localisation des cadastres}

\section{Cadastre A}

Le cadastre $A$ a été localisé au nord de Alpilles suite aux travaux de Gérard Chouquer (Chouquer, 1983) et aux découvertes archéologiques. La trame s'étend entre le Rhône à l'ouest, la Durance au nord, Salon-de-Provence à l'est et la via Aurelia au sud. Le réseau est orienté à $2^{\circ} 30^{\prime} E$ par rapport au nord géographique et s'organise en centuries rectangulaires de 20 par 40 actus de côté (soit 709 et $1418 \mathrm{~m}$ ).

\section{Cadastre B}

Le cadastre $\mathrm{B}$ d'Orange est orienté à $5^{\circ} \mathrm{E}$ par rapport au nord géographique et possède un module basé sur des centuries de 20 actus de côté, soit 708 m. L'extension du cadastre se limite au nord, aux collines de Marsanne, à l'est au bassin de Valréas et au sud au bassin de Carpentras. A l'ouest, le Rhône ne semble pas constituer une limite. La trame encore visible à la confluence de l'Ardèche et du Rhône et est située tout juste au nord de notre zone d'étude.

\section{Cadastre C}

Le cadastre $\mathrm{C}$ est plus lacunaire et a fait l'objet de beaucoup d'hypothèses concernant sa localisation et son orientation. Gérard Chouquer propose une orientation $8^{\circ} \mathrm{W}$ par rapport au nord géographique et un module de 20 actus de côté, mesuré à 710 m. L'assiette de réseau s'étendrait jusqu'à SainteCécile au nord, Bédarrides au sud, Carpentras à l'est et Saint-Geniès-de-Comolas à l'ouest. Le Rhône ne semble pas avoir été, une fois de plus, une limite à l'extension de ce réseau. La limite occidentale de ce réseau se trouve au bord de notre zone d'étude (Chouquer \& Favory, 2001: 217-36). 


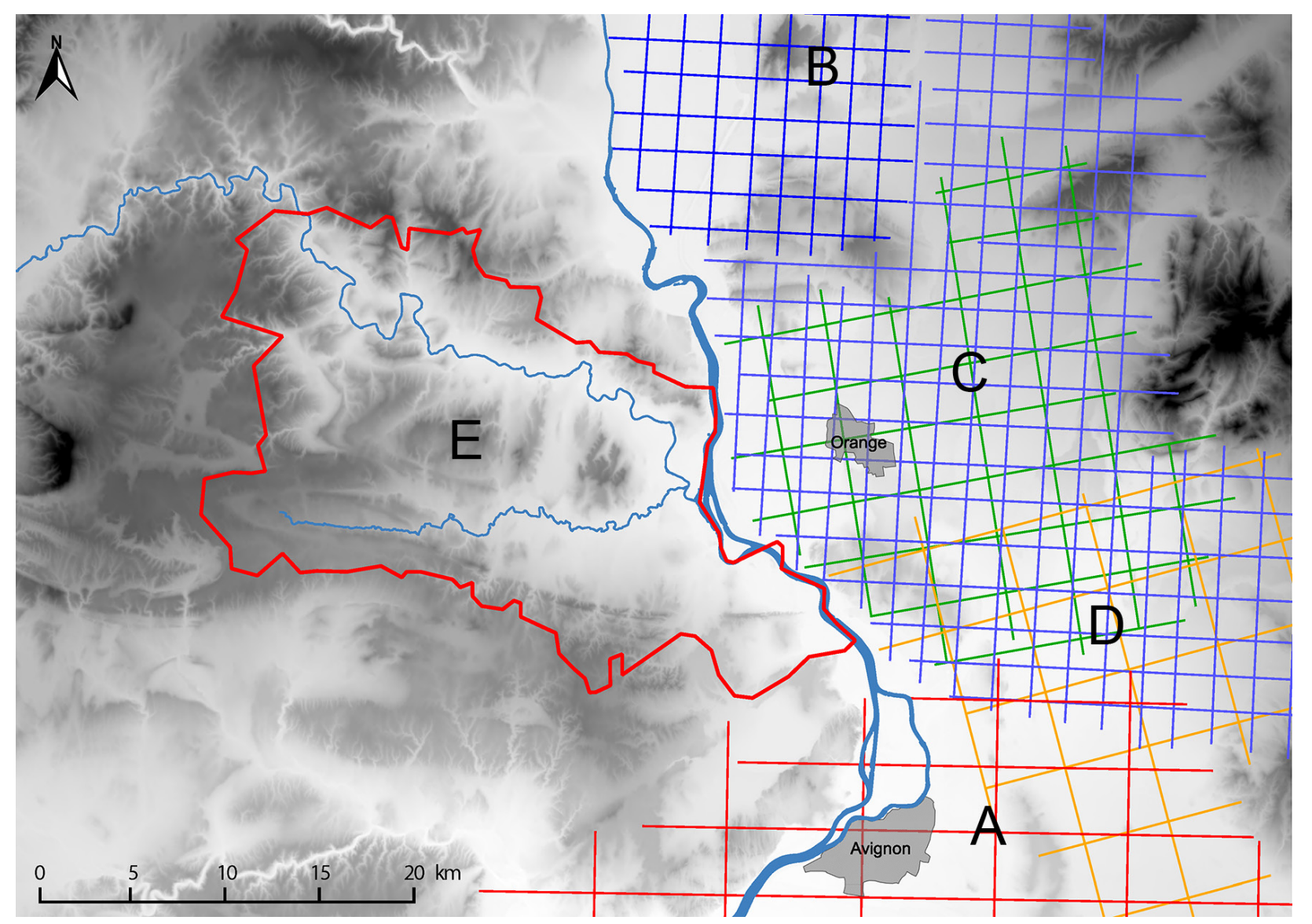

Fig. 2. Cadastres d'Orange et zone d'étude: $A$ - Cadastre $A$ (rouge), B - Cadastre B et B étendu (bleu), C- Cadastre $C$ (vert), $D$ - Cadastre $D$ (jaune), $E$ - Vallées de la Cèze et de la Tave (BDAlti®, CIGN, M. Seguin).

\section{Cadastre D}

Le cadastre $D$ n'est pas représenté par les plans cadastraux, mais mérite d’être mentionné. La trame organise un secteur compris entre Cavaillon et Carpentras. Son module de 705 m est basé sur des côtés de 20 actus et est orienté à $17^{\circ} \mathrm{W}$ par rapport au nord géographique. Cette trame a été identifiée par Mohamed El Hasroufi dans le secteur géographique concerné par notre étude (El Hasroufi, 1994).

Les cadastres $B, C$ et $D$ se trouvent donc en bordure de la zone d'étude choisie et ont des limites encore incertaines au-delà du Rhône (fig. 2).

Cette partie se contente de retracer, dans les grandes lignes, l'évolution des méthodes de détection et d'analyse employées depuis le milieu du XXe siècle. Pour analyse une critique des différentes approches méthodologiques et de leur applicabilité on se référera à l'article d'Hèctor A. Orengo et de Josep M. Palet (Orengo \& Palet, 2010).

\section{Analyse des cartes papier}

Les premiers travaux de détection des parcellaires antiques datent des années 1940 (Poupet, 1987). A cette époque, les moyens de détection étaient très loin des moyens actuels. André Deléage fut un des premiers à identifier une trame antique dans la vallée de la Saône. On dispose aujourd'hui de cartes papier au $1 / 25000$ e, mais ces dernières ne 
peuvent pas constituer le seul support de travail d'une étude parcellaire sérieuse (Assénat, 2006). Elles peuvent permettre de faire une première analyse, mais l'investigation nécessitera par la suite, l'utilisation d'autres supports.

\section{Photographie aérienne}

Dès le début du siècle, la photographie aérienne profite à la recherche des réseaux antiques. Les premières découvertes concernent surtout l'Italie et l'Afrique du Nord où les trames cadastrales sont encore très nettement visibles. Dans les années 1960, Roger Agache interpelle les archéologues sur les possibilités qu'offre la photographie aérienne, mais la recherche archéologique ne prend pas en compte les résultats de ces premières études. Il faudra attendre une exploitation plus poussée des photographies aériennes par filtrage optique pour que la communauté archéologique s'y intéresse.

\section{Filtrage optique}

Au début des années 1980, une nouvelle étape est franchie dans l'interprétation des clichés aériens par l'équipe du Centre de Recherches Archéologiques (USR 1708 du CNRS) associée au Laboratoire d'Optique de l'Université de Besançon (Favory, 1981). En éclairant des clichés IGN avec une lumière cohérente et des filtres directionnels, ils parviennent à extraire les limites parcellaires orthogonales dans une direction choisie. L'intérêt de ce procédé est donc de pouvoir extraire, de manière automatique, les limites parcellaires et le réseau viaire qui sont inclinés dans une même direction. Les premières expérimentations ont porté sur des cadastres déjà connus et repérés, comme ceux de Béziers. Très vite de nombreux cadastres invisibles sur les cartes topographiques furent révélés et l'analyse des résultats montra une superposition de plusieurs cadastres dans certaines régions. Des grilles furent ensuite utilisées pour identifier des cadastres antiques. Cette approche a ensuite été largement critiquée (Leveau, 2005).

\section{Traitement numérique}

Le traitement numérique des images aériennes voit le jour dans les années 1990. Les clichés aériens sont scannés et un logiciel est développé par le Laboratoire d'Optique de Besançon. Les images sont numérisées en niveaux de gris. Le logiciel commence par détecter les bords de contraste significatif. Ils correspondent principalement aux chemins et aux limites parcellaires. Ensuite un filtrage permet d'éliminer le bruit, c'est à dire l'information parasite résiduelle. L'informatique permet d'introduire de nouveaux outils dans l'étude du parcellaire. Notamment l'histogramme des directions qui a été utilisé dans le cadre de cette étude.

\section{Système d'Information Géographique}

En 1987 des travaux sur les cadastres antiques, par traitements informatiques, sont menés à Besançon par une équipe de précurseurs (Vallat, Dodinet \& Leblanc, 1987). Ils renseignent une base de données et calculent les coordonnées théoriques des intersections du réseau orthogonal. Par la suite, l'apparition des ordinateurs personnels et le développement des SIG va permettre plusieurs travaux à Delphes (Clavel-Lévêque \& Tirologos, 2004) et dans la région de Béziers (Monaco \& Clavel-Lévêque, 2004). Paradoxalement, alors que des données vectorielles à grande échelle sont maintenant disponibles (BDParcellaire $®$ ), les données sources continuent à provenir des filtrages optiques de l'imagerie aérienne ou satellitaire. Dans certains cas, les données sont même issues de la vectorisation de cartes papier. Pourtant dès 1993 François Favory écrivait: 'les plans cadastraux sont certainement les plus riches en informations parcellaires. Les plans cadastraux offrent les garanties les plus élevées pour l'approche métrologique' (Favory \& Charraut, 1993: 22-23). Mais à l'époque le plan cadastral informatisé n'existait pas et il était matériellement impossible d'exploiter les documents papier. En effet, l'étude d'une zone de $400 \mathrm{~km}^{2}$ aurait nécessité l'assemblage de plus de 350 feuilles cadastrales au 1/2500e. 


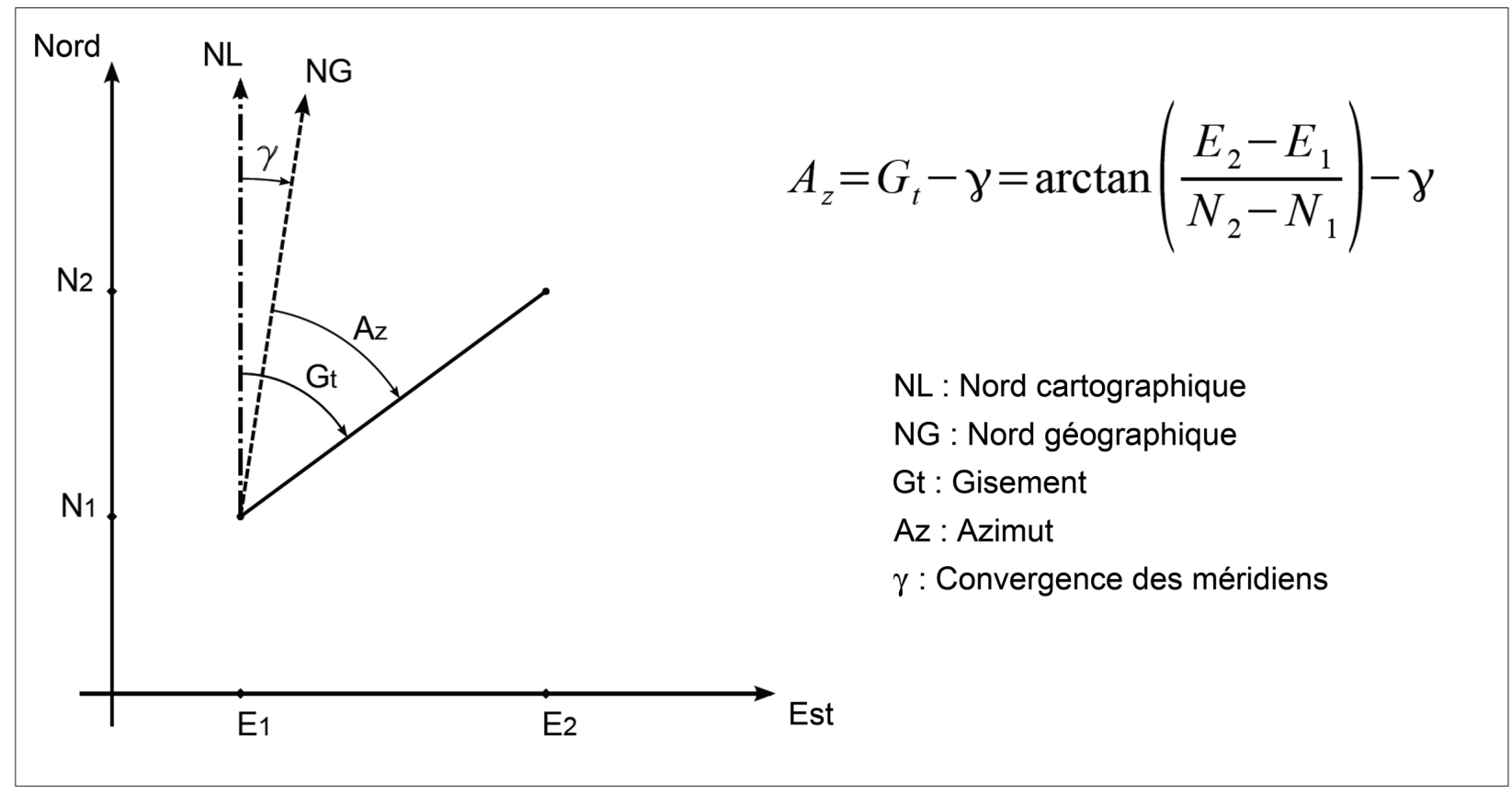

Fig. 3. Calcul de l'azimut (M. Seguin).

\section{Données et outils}

Les données utilisées pour étudier les cadastres antiques dans les basses vallées de la Cèze et de la Tave sont issues de la BDParcellaire ${ }^{\circledR}$ de l'IGN. C'est une donnée homogène obtenue par assemblage du plan cadastral dématérialisé. Elle est géoréférencée et continue pour l'ensemble du territoire français. C'est une description métrique des limites parcellaires au format vectoriel. Elle contient plusieurs données dont la définition surfacique des parcelles cadastrales. C'est ce support qui a été utilisé dans le cadre de cette étude.

Les outils utilisés sont exclusivement des logiciels libres. Les traitements ont été réalisés à l'aide du logiciel GRASS, les données ont été stockées dans une base de données PostgreSQL et l'analyse a été effectuée avec QGIS.

\section{Méthodologie}

Comme on l'a vu, les méthodes pour l'étude des cadastres ont beaucoup évolué. L'outil utilisé dans cette étude est l'histogramme des directions. Il s'agit de calculer l'azimut (fig. 3) de chaque limite parcellaire et d'en tirer un histogramme où le nombre de limites ou leur longueur cumulée sont représentées en fonction de l'azimut (fig. 4). Une structure parcellaire orthogonale produit alors un graphique caractéristique où l'on observe deux pics distants de $90^{\circ}$. Ces pics indiquent l'orientation générale du parcellaire.

L'idée est d'utiliser cet outil, non plus sur des données raster, mais sur un support vectoriel: la BDParcellaire ${ }^{\circledR}$. Les parcelles étant décrites de manière surfacique, il a été nécessaire de décomposer cette information en un ensemble de segments. Ceci permet alors de calculer l'azimut et la longueur de chacun des segments obtenus. Après analyse des histogrammes on peut décider de filtrer le parcellaire selon des orientations choisies avec une plage de tolérance. Dans le cadre de cette étude, les orientations des cadastres d'Orange sont déjà connues. Les parcellaires ont donc été filtrés selon ces orientations. La plage tolérance a été fixée à $3^{\circ}$ à partit de travaux plus anciens et de quelques tests empiriques. 


\begin{tabular}{|l|l|l|l|l|}
\hline & & & & \\
\hline Vallées & Communes & Cadastre B & Cadastre C & Cadastre D \\
\hline \multirow{5}{*}{ Cèze } & Bagnols-sur-Cèze & $\checkmark$ & & \\
\cline { 2 - 5 } & Orsan & $\checkmark$ & $\checkmark$ & $\checkmark$ \\
\hline & Chusclan & $\checkmark$ & $\checkmark$ & \\
\cline { 2 - 5 } & Codolet & $\checkmark$ & $\checkmark$ & $\checkmark$ \\
\hline \multirow{5}{*}{ Tave } & Laudun-l'Ardoise & $\checkmark$ & $\checkmark$ & $\checkmark$ \\
\cline { 2 - 5 } & Saint-Victor-la-Coste & $\checkmark$ & $\checkmark$ & $\checkmark$ \\
\cline { 2 - 5 } & Saint-Laurent-des-Arbres & & $\checkmark$ & $\checkmark$ \\
\cline { 2 - 5 } & Saint-Geniès-de-Comolas & $\checkmark$ & $\checkmark$ & $\checkmark$ \\
\hline & Roquemaure & & $\checkmark$ & $\checkmark$ \\
\hline & Montfaucon & & $\checkmark$ \\
\hline
\end{tabular}

Tab. 1. Synthèse des cadastres détectés

\section{Résultats}

\section{Histogrammes des directions}

Deux histogrammes des directions sont créés pour chaque commune. Le premier représente le nombre de lignes en fonction de l'azimut et le second représente la longueur cumulée des limites pour chaque azimut en fonction de l'azimut. Le choix d'utiliser deux histogrammes fait suite aux premiers tests qui ont été réalisés. On remarque très vite que les résultats peuvent être "bruités" soit par un nombre important de petites limites orthogonales entre elles. C'est le cas de certains lotissements, dont on retrouve la trace dans le graphique. Les résultats peuvent également être "bruités" par de grandes structures linéaires: voies de chemin de fer, autoroute ... et par le remembrement parcellaire qui a pu faire suite à ce type d'aménagement. L'ensemble de ces deux graphiques permet donc de faire une première analyse. Le filtrage permet par la suite de confirmer certaines hypothèses en identifiant sur le plan ces artefacts.

Malgré la présence d'artefacts, les histogrammes indiquent pour chaque commune la présence d'un parcellaire orthonormé. Les valeurs correspondent aux cadastres d'Orange B, C ou D. Pour plusieurs communes on a même la superpo- sition de deux ou trois trames. Le tableau suivant (tab. 1) récapitule les cadastres détectés dans la zone d'étude.

\section{Filtrage des limites parcellaires}

L'analyse des histogrammes a permis de mettre au jour la prédominance de certaines trames orthogonales, mais la présence d'une trame pourrait être occultée par la surabondance d'une autre. Comme nous avons la chance de connaître les orientations théoriques des trames des cadastres d'Orange, nous avons procédé à un filtrage systématique. Il a permis de révéler la présence de parcelles orientées selon des cadastres non détectés par les histogrammes dans les communes de Chusclan et Bagnols-sur- Cèze. Il a également confirmé l'ensemble des observations faites par les histogrammes. Cette phase de filtrage et d'analyse visuelle est indispensable pour analyser les interactions entre les trames cadastrales et les aménagements actuels lvoies ferrées, routes, chemins, drains, ...).

\section{Résultats numériques}

L'ensemble des données concernant les azimuts et la longueur des limites peut être présentées sous forme de tableaux. Ils permettent d'exprimer la présence des trames orthogonales en fonction du pourcentage du nombre de limites conservées 


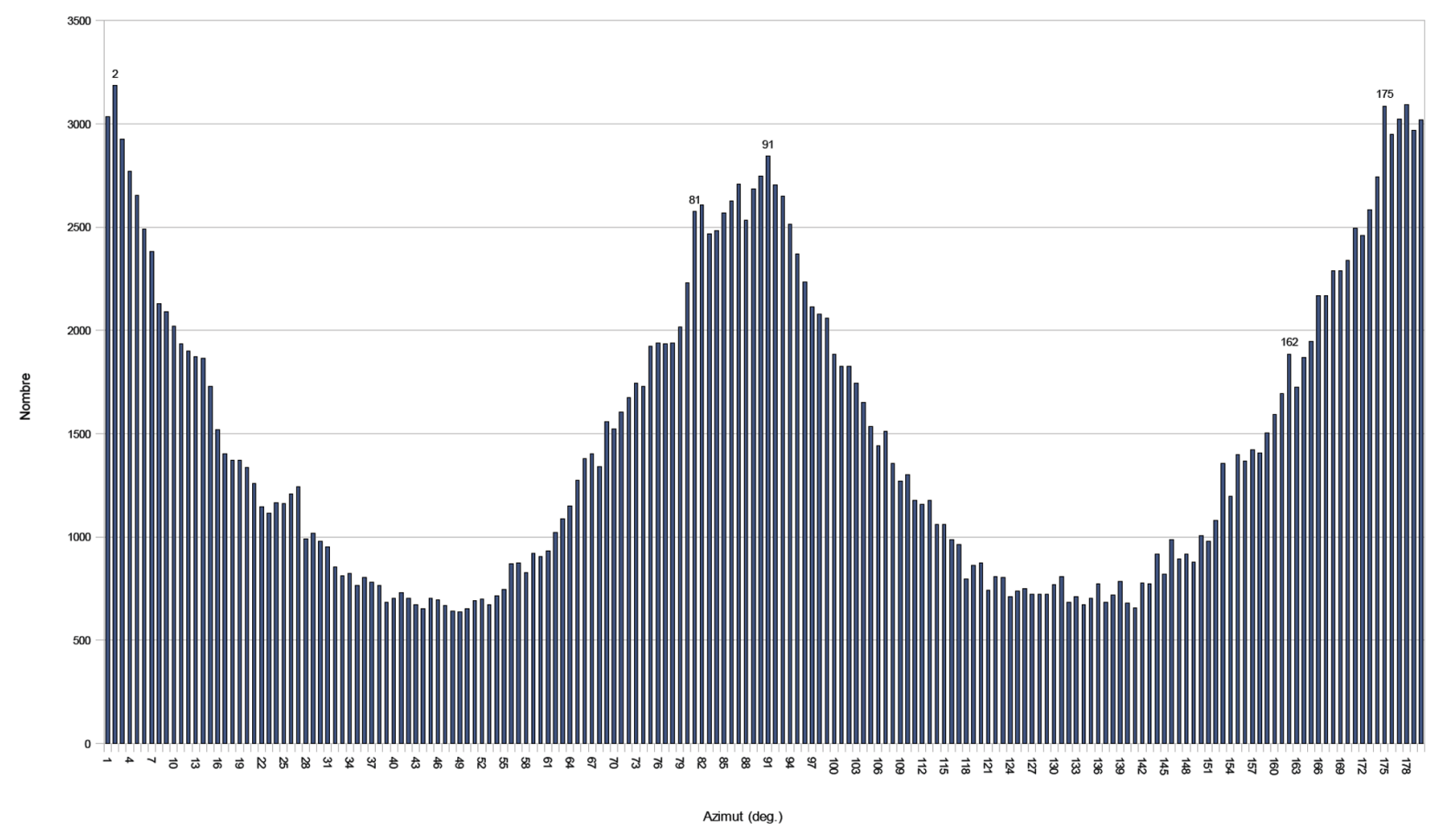

Fig. 4. Histogramme des directions pour les basses vallées de la Cèze et de la Tave (M. Seguin).

et en fonction du pourcentage de longueur cumulée des limites conservées.

Pour pouvoir interpréter les pourcentages exprimés dans ces tableaux, il faut se souvenir que l'on a filtré les limites à $\pm 3^{\circ}$ autour de l'azimut théorique. On a donc sélectionné les valeurs de 14 azimuts sur 180 disponibles. Si on se place dans l'hypothèse d'une distribution aléatoire des azimuts et de la longueur des limites, on devrait donc obtenir le même pourcentage len nombre et en longueur cumuléel pour chaque azimut, soit: 1 $/ 180 * 100=0,56 \%$

Comme on a sélectionné 14 valeurs d'azimuts, les valeurs de pourcentage obtenues sont à comparer à la valeur suivante: $14 * 0,56=7,8 \%$.

\section{Synthèse}

Les résultats obtenus permettent de conclure à la présence des trois cadastres $B, C$ et $D$ dans

les basses vallées de la Cèze et de la Tave. Ces trames sont plus ou moins présentes selon les communes.

Les résultats des histogrammes combinés à une analyse visuelle du parcellaire isocline permettent de cerner l'emprise des différents cadastres antiques.

\section{Cadastre B}

La présence de cette trame dans les basses vallées de la Cèze et de la Tave a depuis longtemps été évoquée par les travaux de Gérard Chouquer et John Peterson (Peterson, 1992). Les résultats obtenus concordent avec les travaux antérieurs et permettent de conclure à l'influence du cadastre B sur l'ensemble des basses vallées de la Cèze et de la Tave. Le parcellaire conservé est extrêmement imprégné par cette trame dans l'ensemble des communes étudiées excepté sur les communes de Codolet et Montfaucon, où les cadastres $\mathrm{C}$ et $\mathrm{D}$ ont pris le dessus. 


\section{Cadastre C}

Cette trame est la plus présente des trois dans le paysage actuel. Elle prédomine surtout dans la vallée de la Tave et sur la rive droite du Rhône. Sa présence est un peu plus ténue sur la partie orientale de la basse vallée de la Cèze où le cadastre $B$ semble s'imposer.

Ces résultats sont un peu paradoxaux. En effet, sur l'ensemble de la vallée du Rhône, le cadastre $C$ est le plus lacunaire et le moins bien connu. La proposition d'assiette du cadastre $\mathrm{C}$ a fait l'objet de nombreuses théories et l'hypothèse actuellement admise est celle de Gérard Chouquer.

\section{Cadastre D}

Contrairement aux cadastres B et $\mathrm{C}$, cette trame n'est pas connue au travers de documents cadastraux, mais sa présence dans une partie de la vallée du Rhône est maintenant communément admise (Leveau, 2010: 137-38). Les résultats obtenus concordent avec ceux de Mohamed El Hasroufi (El Hasroufi, 1994) en ce qui concerne sa présence sur les communes de Montfaucon, Roquemaure et Saint-Geniès-de-Comolas. On peut même étendre sa présence aux communes de Laudun-L'Ardoise, Codolet et Chusclan. Il semblerait que cette trame n'ait pas pénétré dans les basses vallées de la Cèze et de la Tave. Sa présence est principalement détectée sur une bande d'environ quatre kilomètres de large, le long de la rive droite du Rhône.

\section{Conclusion}

Cette étude a montré la possibilité d'étudier le parcellaire antique à l'aide d'un système d'information géographique et de la BDParcellaire ${ }^{\circledR}$ de l'IGN. Cette méthode reste à ce jour inédite, malgré sa simplicité de mise en œuvre, en comparaison au filtrage optique ou au traitement numérique des clichés aériens. L'intérêt d'utiliser des données parcellaires tient également au fait que l’on a à disposition des informations sur le parcellaire visible et des informations sur des limites parcellaires non matérialisées dans le paysage.

En ce qui concerne les résultats, ces derniers concordent en tout point avec ceux des filtrages optiques réalisés par Gérard Chouquer et François Favory. Avec les données vectorielles nous avons même pu réaliser une étude plus fine au niveau de l'organisation des centres historiques des villages, ce qui est impossible à réaliser avec des données cartographiques classiques ou avec des clichés aériens.

Ces résultats très encourageants nous pousseront à porter l'étude sur l'ensemble des vallées de la Cèze et de la Tave, en complément de l'étude réalisée sur les parties basses de ces vallées.

La mise à disposition gratuite des données du RGE $®$ aux établissements d'enseignement et aux organismes de recherche devrait permettre le développement de ce type d'étude.

La combinaison des données issues de la BDParcellaire ${ }^{\circledR}$ avec celles d'autres données topographiques ouvre la voie à une analyse archéomorphologique plus détaillée. L'étude pourra également être complétée par l'exploitation des données issues des prospections pédestres et des fouilles archéologiques. Enfin, le développement récent de l'archéologie préventive dans le nordest du département du Gard ouvre de nouvelles perspectives à cette étude.

\section{References}

Alix S, 2008: Des garrigues au Rhône; occupation du sol et dynamiques de peuplement dans le nord-est du Gard (rapport annuel de PCR), SRA Languedoc-Roussillon, Montpellier.

Assénat M, 2006: Cadastres et romanisation dans la cité antique de Nîmes, Revue archéologique de Narbonnaise, 36

Chouquer G, 1983: Localisation et extension géographique des cadastres affichés à Orange, in Clavel-Leveque M (ed.), Cadastres et espace rural. Approches et réalités antiques: 275-95. CNRS, Paris. Chouquer, G \& F Favory 2001: L'arpentage romain. His- 
toire des textes, droit, techniques, Errance, Paris.

Clavel-Lévêque M \& G Tirologos 2004: Analyse spatiale, archéologie des paysages et centuriation, application des méthodes SIG: 1 - La restitution d'un parcellaire et la modélisation des lots: le territoire sacré de Delphes, Dialogues d'histoire ancienne, 30, 183-85.

El Hasroufi M, 1994: Cadastres, territoires et cités. Le réseau $D$ d'Orange: un cadastre romano-indigène inédit, Dialogues d'histoire ancienne, 20, 360-74.

Favory F, 1981: Détection des cadastres antiques par filtrage optique: Gaule et Campanie, Mélanges de l'Ecole française de Rome, 92, 347-86.

Favory F \& D Charraut 1993: De la carte topographique à l'analyse d'images: méthodologie

de l'identification des limitations antiques, Revue archéologique de Narbonnaise, 26, 19-56.

Leveau P, 2005: L'archéologie du paysage et l'antiquité classique, Agri Centuriati: an International Journal of Landscape Archaeology, 2, 9-24.

Leveau P, 2010: La centuriation des territoires des cités romaines d'Arles (Arelate) et d'Aix-en-Provence (Aquae Sextiae). Un retour historiographique, Revue archéologique de Narbonnaise, 43, 129-54.

Monaco M \& M Clavel-Lévêque 2004: Analyse spatiale, archéologie des paysages et centuriation, application des méthodes SIG: 2 - La modélisation d'un paysage centurié: le Sud Biterrois ; 3 - La reconstitution d'un paysage antique: l'ager Campanus, Dialogues d'histoire ancienne, 30, 186-200.

Orengo HA \& JM Palet 2010: Methodological insights into the study of centuriated field systems: a landscape archaeology perspective, Agri centuriati: an International Journal of Landscape Archaeology. 6(2009), 171-85.

Peterson JWM, 1992: Computer aided projection of part of the Orange $B$ cadastre to Cèze valley, Dialogues d'histoire ancienne, 18, 169-76.

Piganiol A, 1962: Les documents cadastraux de la colonie romaine d'Orange, Éd. du Centre national de la recherche scientifique, Paris.

Poupet P, 1987: Convergence des recherches sur les cadastres, les parcellaires et les terroirs, Revue archéologique de Narbonnaise, 26, 11-17.

Seguin M, 2010: Le SIG comme outil de détection et d'analyse des parcellaires antiques : le cas des basses vallées de la Cèze et de la Tave (mémoire de travail de fin d'étude). Conservatoire National des Arts et Métiers, Ecole Supérieure des Géomètres Topographes, Le Mans.

Vallat J-P, M Dodinet \& J Leblanc 1987: Utilisation de moyens informatiques en archéologie du paysage, Dialogues d'histoire ancienne, 13(1), 315-55. 